\title{
Internet, l'autoroute et les chemins de traverse
}

\section{Nicole Chenik}

\section{OpenEdition \\ Journals}

Édition électronique

URL : http://journals.openedition.org/asp/3951

DOI : 10.4000/asp.3951

ISSN : 2108-6354

\section{Éditeur}

Groupe d'étude et de recherche en anglais de spécialité

\section{Édition imprimée}

Date de publication : 1 décembre 1995

Pagination : 359-379

ISSN : 1246-8185

\section{Référence électronique}

Nicole Chenik, «Internet, l'autoroute et les chemins de traverse », ASp [En ligne], 7-10 | 1995, mis en ligne le 15 novembre 2013, consulté le 07 mai 2019. URL : http://journals.openedition.org/asp/3951 ; DOI : 10.4000/asp.3951

Ce document a été généré automatiquement le 7 mai 2019.

Tous droits réservés 


\title{
Internet, l'autoroute et les chemins de traverse
}

\author{
Nicole Chenik
}

1 Si les autoroutes électroniques ou inforoutes, qui font l'objet du récent rapport Théry, se font encore attendre, des outils existent déjà qui en donnent un avant-goût. L'Internet, ce réseau des réseaux, connecte maintenant de vingt à trente millions d'ordinateurs dans plus de soixante-quinze pays chaque jour, sur les deux cents pays connectés. L'explosion de la communication électronique est permise par la technologie. Un micro-ordinateur et un modem suffisent maintenant pour explorer les réseaux. Les modems, qui permettent de transmettre des informations sur une ligne téléphonique ont baissé de prix, leur vitesse est plus élevée, le volume transporté augmente donc avec une capacité accrue à transmettre texte, son et images.

2 La France se relie à l'Internet par l'intermédiaire de RENATER (Réseau National de Télécommunications pour 1a Technologie, l'Enseignement et la Recherche) dont la mise en place date de juin 1992 et dont l'objectif est d'offrir à l'ensemble des établissements d'enseignement supérieur et aux équipes de recherche universitaires ou industrielles une infrastructure de communication nationale et internationale à haut débit, ainsi que des services nationaux de messagerie. Les réseaux de campus sont reliés aux réseaux régionaux, eux-mêmes reliés au réseau national et international. La plupart des universités françaises sont reliées à RENATER, mais de grandes disparités existent entre les départements scientifiques et littéraires.

D'un point de vue historique, l'ancêtre de l'Internet est le réseau ARPANET, qui avait été mis en place dans les années soixante par la Défense américaine pour la diffusion interne de l'information. Il fallut attendre 1989 pour que le CERN, sous l'impulsion de Tim Berners-Lee, mette en service un réseau qui fonctionna d'abord en interne. La présentation officielle eut lieu en 1991. C'est également en 1991 que fut organisée la première conférence HYPERTEXT 91. Le système fut développé ensuite et prit véritablement son essor à partir de 1992/93. 
4 On utilise communément le terme d'Internet pour faire référence à l'ensemble des ordinateurs qui sont reliés par connexions informatiques, alors que le terme World Wide Web (WWW, W3, The WEB) fait davantage référence à l'ensemble de l'information, à l'espace de savoir qui est disponible sur le réseau. L'Internet n'a pas à proprement parler de gestion centralisée bien que le Internet Architecture Board (IAB) définisse les normes et alloue les adresses et que le Internet Engineering Task Force (IETF) s'occupe des problèmes techniques et de sécurité. Des utilisateurs mettent des documents à la disposition d'autres utilisateurs. Ces utilisateurs peuvent aussi bien être des administrations gouvernementales, des musées, des bibliothèques, que des unités plus modestes comme les universités, les centres de recherche, voire des individus.

5 Avoir accès à l'Internet donne diverses possibilités : l'accès à des ressources diverses (y compris les banques de données et les catalogues de bibliothèques); l'utilisation du courrier électronique (email); l'accès à des forums de discussion (en temps réel ou en temps différé) ; l'accès à des nouvelles sur l'organisation et les nouveaux services du réseau; la possibilité de transférer et de ramener à soi des fichiers, et de les imprimer éventuellement.

6 L'Internet a connu et connaît une croissance tout à fait extraordinaire. En un an, le trafic sur WWW, mesuré en octets, a été multiplié par 187. On compte environ 12000 sites connectés, et il y en a de nouveaux chaque jour. On dénombre environ un million d'utilisateurs nouveaux par mois. Cette croissance phénoménale et l'augmentation spectaculaire en 1993/94 sont dues au fait qu'il existe maintenant des interfaces graphiques qui rendent beaucoup plus aisée et conviviale la navigation, et inutiles les commandes Unix à l'aspect parfois rébarbatif pour les non-informaticiens.

\section{Outils}

\subsection{Outils de navigation}

7 Les outils de navigation sont: WWW (World Wide Web); MOSAIC et X MOSAIC (National Center for Supercomputing Applications, University of Illinois) ; NETSCAPE; GNN (Global Network Navigator) qui est un magazine électronique auquel il faut s'inscrire, mais dont l'accès est gratuit; ARCHIE (FTP search service); VERONICA (Gopher search service) qui permettent des recherches spécialisées (par mots clés, par l'intermédiaire de FTP [File Transfer Protocol] ou par Gopher). Ces outils sont des interfaces de type hypertexte.

\subsection{Fonctionnement}

\subsubsection{Architecture}

8 Ces outils sont organisés selon une architecture client-serveur. Sans vouloir aller plus avant dans des détails techniques, on dira simplement qu'il s'agit d'un programme (client) qui demande un document et d'un autre programme (serveur) qui répond à la demande et envoie ce document. 


\subsubsection{Formats}

Pour communiquer entre eux, les réseaux ont besoin d'un langage commun, qui permet de transférer la demande. Il s'agit de HyperText Transfer Protocol (HTTP). Pour envoyer le document hypermédia, le créer, le reconnaître, on a aussi besoin d'un standard, qui est HyperText Markup Language (HTML). Assez proche du Standard General Markup Language (SGML), il permet de séparer l'information contenue de la présentation du document (ce qui permet de changer éventuellement la présentation de l'information). La version HTML+ est en cours de développement.

\subsubsection{Adresses ou URL (Uniform Resource Locator)}

10 Tout fichier peut être représenté par une URL qui obéit à une syntaxe particulière et se compose de plusieurs parties : une première série de lettres donne une indication sur le mode d'accès, par exemple http ou ftp (File Transfer Protocol, pour un fichier), news (sur USENET), ou gopher, puis: //qui est suivi de l'adresse de l'ordinateur, et du nom des fichiers, ainsi que l'endroit du fichier qu'il faut ouvrir, séparés par des points et des traits obliques.

11 En allant à l'adresse <http://www.honolulu.hawaii.edu>, on a donc accès aux informations contenues dans le fichier correspondant «edu» situé à Hawaï, sur le serveur « Honolulu [Honolulu Community College], en utilisant WWW, par HyperText Transfer Protocol.

\subsubsection{Que peut-on faire à partir d'un écran ?}

12 Mon utilisation personnelle est celle de X MOSAIC et de NETSCAPE, qui sont très conviviaux, presque user cudly, en particulier NETSCAPE.

13 On peut avoir accès à un texte (à une ou plusieurs "pages»), à des liens hypertextes (certains mots sont soulignés, ou en gras, couleur ou italiques, ou bien des icônes sont présentes). En cliquant sur ces indications, on a alors accès à des éléments complémentaires; accès à un document sonore, à une image fixe, à des images animées vidéo (en activant l'icône correspondante). À tout moment, on a une indication de l'endroit où on est, et la possibilité d'enregistrer cette adresse pour référence ultérieure.

14 Il est possible de se déplacer dans le texte : en avant, en arrière, ou de décider d'aller ailleurs (avec les indications GO TO, puis l'adresse voulue/OPEN).

15 Il est possible de laisser, par le biais des bookmarks, sortes de marque-pages, une trace de son passage, comme on marquerait la page d'un livre que l'on désire relire. On peut donc constituer un répertoire d'adresses où on peut revenir ultérieurement. En activant ADD BOOKMARK, on ajoute l'adresse voulue à 1a liste en cours de constitution. Ceci établit un lien hypertexte qui sera ensuite réactivé en cliquant sur cette adresse si on désire consulter le même service.

On peut afficher les traces des endroits ainsi visités, savoir quel a été le cheminement et revenir à un point de la recherche qui nous a intéressés.

Il est possible, non seulement de consulter en ligne les informations, mais aussi de les ramener à soi en quelque sorte, de décharger les fichiers (ainsi que les images) soit en tant que fichiers auxquels on peut ensuite avoir accès (sur disque dur ou sur le réseau), 
soit par impression de leur contenu (PRINT). Lorsque le transfert du fichier est en cours, on peut visualiser la progression du processus à l'écran, ce qui est très pratique en cas de chargement très long. On a également l'option d'arrêter le chargement.

\subsubsection{Homepages} utilisateurs de l'Internet. Il faut créer sa propre « HOMEPAGE », qui est le point de départ pour rassembler les informations que l'on désire mettre sur l'Internet, les organiser sous forme de menus et de liens hypertextes que l'on désire établir. C'est d'ailleurs ce qui a été fait à l'Université de Paris 9 Dauphine, IUP MIAGE, où un petit serveur existe maintenant ${ }^{1}$.

Les projets sont d'inclure dans ce serveur un certain nombre de documents pertinents pour les langues. Au niveau de l'université, une des tâches de la cellule multimédia qui se met en place sera d'établir un serveur Dauphine sur l'Internet, où on trouve déjà pour la France non seulement le Ministère de la Culture, mais aussi la plupart des grandes écoles françaises, des centres de recherche, essentiellement informatiques, et autres services (environ 260 dont la liste est accessible sur Netscape, avec possibilité d'entrer un nouveau nom de serveur, et de recherche par mot clé).

\section{Utilisations : un réservoir d'informations}

Le propre de l'Internet et sa richesse principale sont bien sûr la somme considérable des informations disponibles, mais surtout la facilité avec laquelle il est possible de naviguer dans cette masse énorme. Il est donc à la fois paradoxal et frustrant de vouloir donner ici une typologie relativement organisée des divers types d'utilisation. La fascination que peut exercer l'Internet sur son utilisateur provient justement de la rapidité et de la simplicité avec laquelle ce dernier va pouvoir passer de la Library of Congress à l'université de San Diego, de Shakespeare au Spiegel, d'un plan de Paris aux manuscrits de la Mer morte ou à un scénario de Pulp Fiction, de la photo de Socks, le chat de Bill Clinton, qui fait très officiellement partie de la famille lorsqu'on accède au serveur de la MaisonBlanche, au texte officiel du traité de Maastricht, et les exemples sont légion. La démonstration en ligne de NCSA MOSAIC donne la liste suivante: US High Performance Computing and Communications, University of Illinois at Urbana-Champaign, Arts and Humanities--Education and Research, Government and the Clinton/Gore administration, Industry, business and publishing, NASA and Government Laboratories, Science, Technical and Professional Information, Other prototypical Hypermedia applications, Experimental/Advanced Hypermedia demos, Other Internet Information resources.

21 Les ressources sont si vastes que des outils de navigation comme GNN proposent différents guides. La page d'accueil permet de sélectionner: The Whole Internet Catalogue, The GNN Business pages, GNN Netnews, Special interest Publications, Traveler's Center, Personal Finance Center, I-Media Center (discuss the design, technologies and strategies of publishing on the Internet), etc.

The Best of the Net propose les rubriques suivantes, avec pour chacune un bref descriptif du contenu: Artserve (Base de données sur l'histoire de l'art, architecture et sculpture); The currency converter (taux de change et conversion d'une devise à l'autre); Edupage Newsletter/EDUCOM (education et nouvelles technologies); International Teletimes (magazine d'intérêt général) ; Internet underground Music archive; New Zealand information; 
The paleontologty server; Science fiction resource guide; Taxing times; Us census information server; Hypertext USENET FAQs (Frequently Asked Questions); Xerox Park Map viewer; Howard Reingold, first citizen on the Net (on virtual communities).

Si l'on veut cependant dresser une typologie, et non une liste, des ressources, elle pourrait être la suivante: outils, textes électroniques, données pour logiciels pédagogiques, accès aux homepages d'autres universités, forums (discussion lists) en temps réel ou en différé, newsletters, banques de données, ressources diverses et cours.

\subsection{Outils}

Il s'agit essentiellement de logiciels, pour PC ou pour Mac, qui existent en shareware (ou partagiciel, si l'on préfère le néologisme à la version originale), et que l'on peut décharger directement à partir d'une adresse. On peut citer le programme de concordance TACT 2, par exemple, ou encore des polices de caractères particulières, comme pour le russe.

\subsection{Textes électroniques}

De très loin, c'est la ressource la plus variée, et les indications mentionnées ici ne sont de toute évidence qu'une infime partie de l'ensemble.

\section{Encyclopédies, dictionnaires.}

Il peut s'agir des outils habituels, mais du point de vue du linguiste, des approches différentes ont leur intérêt. C'est ainsi que l'on peut avoir accès, par le serveur de Carnagie Mellon, à un dictionnaire anglais-allemand/allemand-anglais, ou à des dictionnaires de langues plus exotiques. On trouve par exemple College slang dictionary, Esperanto-English dictionary, glossary of computer terms in Vietnamese, resources for adult learners of Welsh.

\section{Journaux}

De plus en plus de journaux rendent accessible le contenu des informations en ligne, ce qu'on baptise maintenant l'information électronique, digital ink. C'est ainsi que l'on peut avoir accès au Washington Post, Time Magazine, San Francisco Free Press, ou au Spiegel. CNN diffuse également des bulletins en ligne, ainsi que les agences de type Reuter. Certains magazines ont un accès limité, par exemple le mythique Wired, organe de la mouvance " cyberculture ", mais peu sont payants. Pour avoir accès à Wired, par exemple, il suffit d'être inscrit sur leur liste de courrier électronique et l'accès est ensuite gratuit.

\section{Littérature}

Sous forme de textes électroniques, il est maintenant possible d'avoir accès à de très vastes corpus. L'accès est donc plus facile que les ressources de l'oxford Text Archive par exemple ou celle de la Text Encoding Initiative. Certains textes permettent des recherches par références croisées, d'autres simplement de décharger le texte pour pouvoir ensuite l'utiliser à d'autres fins, dans des programmes de concordance, par exemple. C'est ainsi que l'on peut avoir accès à la totalité des œuvres de Shakespeare, avec accès par mots clés, ainsi qu'à la filmographie, ou à un Shakespeare illustrated, où ont été réunis des tableaux directement reliés aux pièces de théâtre, ainsi que des commentaires.

Parmi d'autres titres, citons Alice in Wonderland avec la totalité des illustrations du texte original, qu'une imprimante de bonne qualité (postscript) restitue de manière assez satisfaisante. 
Deux sources essentielles de textes électroniques sont The English Server at Carnagie Mellon, et Wiretap, où la nature des textes est très variée (voir annexe).

Bien qu'il ne donne pas accès aux textes électroniques à proprement parler, signalons le serveur < http://www.h-net.org/ clc/etext/CETH.html> du Center for Electronic Texts in the Humanities (Rutgers and Princeton Universities) qui recense les centres s'occupant essentiellement de l'étude des textes électroniques (The Directory of Electronic Text Centers) ${ }^{2}$ . Ceci peut être le premier pas vers une recherche plus spécialisée.

Toute adresse contient bien entendu du texte, sous forme électronique, et ces ressources utilisables à divers titres sont abordées à la section 2.9.

\subsection{Données pour logiciels pédagogiques}

L'adresse est celle de CELIA (Computer Enhanced Language Instruction Archive), qui met à disposition sur l'Internet plusieurs ensembles de données destinées à fonctionner avec des logiciels pédagogiques pour Mac ou PC. Il y a par exemple 34 séries de données pour Mac à utiliser avec des logiciels tels que CHOICEMASTER, GAPMASTER, TESTMASTER, et des exercices de vocabulaire.

\subsection{Accès aux Homepages d'autres universités}

L'utilisation que l'on peut avoir à partir de la homepage d'une université présente sur l'Internet est multiple.

On peut avoir accès à une présentation des campus, des cours offerts par l'université, des équipements, sorte de brochure informatisée illustrée (plan, images) destinée aux étudiants qui désirent s'inscrire. Honolulu Community College a été un des premiers à figurer sur l'Internet et ce serveur est très riche.

L'université s'arrête rarement là. Sont généralement accessibles les listes des centres de recherche, publications spécialisées, listes des enseignants et chercheurs, qui eux aussi décident des informations qu'ils désirent voir figurer sous leur nom. Les pratiques sont diverses, de la notice très sommaire, en passant par les publications détaillées et activités de recherche, sans oublier très souvent la photo de la personne quand ce n'est pas le paysage vu de la fenêtre du bureau. Les coordonnées de personnes ressources ou enseignants chercheurs sont ainsi directement à la disposition des collègues bien sûr, accessibles internationalement, et à la disposition des étudiants chercheurs qui peuvent prendre contact directement avec les meilleurs spécialistes de leur domaine. Le serveur de l'université de Berkeley est très représentatif à cet égard.

Une université comme Wuppertal en Allemagne a aussi fait le choix de présenter son menu dans trois langues différentes, et de créer des liens avec d'autres sites Internet. C'est ainsi que directement à partir de la homepage de l'Université de Wuppertal, on a accès au serveur de la Maison-Blanche ou au Spiegel, ou encore au film des Beatles, A Hard Day's Night.

Ces ressources sont aisément accessibles aux étudiants de langue, ce qui leur évite de faire les recherches eux-mêmes (et diminue le temps de connexion au réseau et donc les coûts), sans les empêcher pour autant d'explorer d'autres possibilités. De plus, il y a souvent sur les serveurs des "embouteillages", et il est parfois frustrant de se voir refuser l'accès. La contrepartie est bien sûr qu'à décharger trop de données, on risque 
aussi de bloquer très vite son propre serveur au niveau local, et ce type de pratique nécessite donc des moyens informatiques appropriés.

Tout un travail a été entrepris à l'Université de Wuppertal par le biais de la création d'un WWW module par les enseignants de langue, comme l'a présenté A. Eck dans sa communication Hypertext: The Global Touch, lors de la conférence EuroCALL 94 de Karlsruhe.

De plus et à terme, les résultats de projets comme STACCATO $^{3}$ vont permettre l'accès en ligne aux ressources des centres de langue européens de l'enseignement supérieur et devraient déboucher sur des échanges en ligne de matériel pédagogique (textes, mais aussi son et vidéo).

\subsection{Discussion lists}

Elles sont nombreuses, et les messages que l'on envoie sont diffusés à l'ensemble des membres inscrits qui peuvent ainsi réagir, envoyer une réponse, un commentaire, une suggestion. Les forums (discussion lists) sont le plus souvent supervisés par une personne responsable (moderator) qui veille à la nature des propos échangés. Les échanges sont bien sûr de nature diverse, organisés par thèmes (par exemple contribution à la réflexion sur "Cyberspace and the American Dream, the New Electronic Frontier ») ou d'ordre technique, où un utilisateur à l'autre bout du monde vous indiquera comment lui a su résoudre tel problème technique dans l'utilisation d'un logiciel. Les échanges entre scientifiques du monde entier passent de plus en plus par l'Internet, et les listes se structurent par centres d'intérêt ${ }^{4}$.

Pour l'enseignement des langues et les nouvelles technologies, citons, pas encore sur l'Internet, mais sur JANET, le forum du nom de UNTILL (Using New Technologies In Language Learning).

Les forums peuvent aussi être en temps réel, selon le principe du courrier électronique. Il va de soi que les forums par domaine de spécialité sont autant de sources de textes spécialisés pour nos étudiants dits non spécialistes.

\subsection{Newsletters}

Des associations publient directement leurs bulletins d'information, dates de conférences, séminaires etc. sur l'Internet dans différents domaines. Pour les langues, citons ReCALL Newsletter organe du Computers in Teaching Initiative Centre for Modern Languages, ainsi que toutes les informations sur Information Technology Training Initiative. (ITTI)

Pour ceux d'entre nous qui s'intéressent aussi aux sciences humaines, le Computers in Teaching Initiative Centre for Textual Studies d'Oxford vient également de mettre en place le serveur <http://www.ox.ac.uk/depts/humanities/> sur lequel on peut trouver la version électronique du Resources Guide, et prochainement le contenu de la lettre d'information Computers and Texts publiée par le CTI d'Oxford.

\subsection{Banques de données}

L'accès peut être soit public et gratuit, soit payant. Il peut s'agir de catalogues de bibliothèques, de références bibliographiques ou de publications en texte intégral. 

apprentissage par la découverte. On ne décrira ici brièvement que quelques adresses parmi les plus célèbres et les plus pertinentes aussi d'un point de vue civilisationnel.

\section{The Whitehouse}

La page d'accueil représente une photo de la Maison-Blanche avec les icônes suivantes: Executive branch, The First family, Tours, President's Welcome message, Guest book, Vice President's welcome message, Comments, Publications, What's new?

L'Interactive Citizen's Handbook permet aux particuliers de remplir des formulaires où ils peuvent exprimer leur opinion sur tel ou tel aspect de la politique.

3 L'icône What's New permet d'avoir plus d'information sur National Information Infrastructure Progress Report, Recently opened federal agency Internet services, Vice president Al Gore's favorite political cartoons.

Les publications de la Maison-Blanche sont particulièrement intéressantes pour les américanistes. Elles incluent: Daily press releases, Topical releases, Major documents (ex: Health Security Act), National documents (GATT), Historical documents (The Declaration of Independence, The United States Constitution) ainsi que la possibilité de s'abonner par courrier électronique aux publications de la Maison-Blanche.

The CIA/FBI

Ces serveurs sont particulièrement riches, et on y trouve aussi bien des informations politiques, géographiques, et démographiques, que les activités du FBI en ce qui concerne les recherches et enquêtes sur les serial killers.

La homepage de la CIA propose The World Factbook 1994, Factbook on Intelligence, avec des données d'une précision extrême sur tous les pays du monde.

The Library of Congress

Le serveur, un des plus visités, propose: About the Library of Congress WWW, Exhibits (Recents selections from Library of Congress exhibits), American Memory (Selected collections from the Library of Congress, "primary source and archival material relating to American culture and history"), Country studies (Area handbooks), Global Electronic Library (Other WWW resources by subjects), LC Marvel (Gopher-based Campus-wide Information System), LOCIS (Search Online Catalogs and databases). 

School of Law par exemple, où sont accessibles de nombreux textes juridiques ${ }^{7}$. Le serveur WIRETAP est également une source très riche dans des domaines variés ${ }^{8}$. On trouve des textes sur l'espace sur le serveur de la NASA, et un grand nombre sur les serveurs des laboratoires informatiques. Pour la médecine, la dernière prouesse est le projet The Visible $M^{9}{ }^{9}$, qui rend disponibles des images numérisées extrêmement complètes d'un corps humain, nécessitant pas moins de 15 gigaoctets d'espace de stockage (et deux semaines de consultation sur Internet pour avoir accès à la totalité des données.)

Il est sûr que d'avoir des ressources disponibles incite à l'exploration et aiguise la curiosité. Tout un aspect ludique est également présent, et il est difficile de résister à des adresses clin d'œil, comme Pizza Hut, le Guide du Routard, ou la visite guidée de Paris à partir du serveur de l'université de San Diego. Ce dernier propose, avec illustrations, Visitor's center, Metro RER, Monuments, Museums, Special Expositions, Scenes from Paris, Cafés, Calendar, Paris Guest book, Schools, Faculties \& institutes in and around Paris, Paris Glossary, Other Links about Paris and France, Selected bibliography of Paris, etc.

\subsection{Cours}

65 Des cours en tant que tels commencent à être disponibles. Par exemple, une English composition class, ou bien Technical writing. qui propose a study of the principles, processes and products which constitute technical writing. Sont donnés en ligne les objectifs, les méthodes de travail, les conseils sur l'utilisation des ressources informatiques à la disposition des étudiants, des explications pratiques sur les dates des examens, l'évaluation, la notation, le travail à faire, le programme du cours. Une partie du travail est effectué en ligne, le reste en cours. Les devoirs sont récupérés directement par l'enseignant sur le compte informatique des étudiants à une date fixée.

On trouve aussi des cours de latin (Latin on the Net, University of Pennsylvania College of general studies), l'université de Southampton propose des cours en Information Engineering, 
tandis qu'aux États-Unis s'organise la première université entièrement électronique. Fondée par l'astrophysicien Joseph Wang, à Austin, Texas, The Globewide Network Academy (Le Monde de l'éducation 1994), sans salles de cours, veut être un établissement à but non lucratif, ouvert à tous sans hiérarchie ni administration. Elle fonctionne grâce au travail de bénévoles, possède sa bibliothèque et son réseau d'experts. Elle enregistre 3000 demandes par jour et les cours concernent pour l'instant les langues vivantes, l'informatique, l'économie et les sciences naturelles.

L'écho arrive d'ailleurs jusqu'en Europe puisqu'un récent séminaire (1994) organisé par le Directorate General DGXIII de la Commission Européenne incluait une communication du Dr. Chris Hutchinson (Kingston University) intitulée New access to a University Education: The Global University.

\section{Internet, échanges et exploration}

L'Internet, c'est la profusion de l'information. Il peut y avoir un certain vertige de la "Cyberia ». Il est utile, semble-t-il, de faire la distinction entre données et information. S'il est éminemment facile d'avoir accès à une quantité faramineuse de données, il n'est toutefois pas toujours aisé d'avoir accès à une information qui soit pertinente par rapport à sa recherche, d'où un travail nécessaire sur l'organisation des données pour qu'elles puissent aisément se transformer en informations directement utiles. Des logiciels sont en train d'éclore, qui se chargent du tri des données, et des recherches en informatique documentaire sont en cours pour précisément trouver et définir les meilleurs moyens de gérer ce vaste réservoir d'information si aisément accessible.

L'Internet est un réservoir inépuisable de ressources authentiques de nature fort différente, pouvant mener à des activités diverses. L'essentiel de l'information est en anglais, ce dont les anglicistes se réjouissent, puisque cela donne nécessairement à lire aux étudiants qui ont alors un but très concret lors de certaines activités de lecture et de compréhension écrite, lesquelles ont parfois été délaissées au profit d'activités de communication. Mais les collègues germanistes devraient trouver intéressante la visite de la ville de Passau pendant la dernière guerre, les ressources en espagnol ont également fait leur apparition, ainsi que des serveurs en Russie, qui, même si la rédaction ne s'y fait pas directement en russe à cause des problèmes que présente l'alphabet cyrillique, sont autant de fenêtres culturelles. Les étudiants en informatique, par exemple, utilisent assez facilement les ressources Internet pour leurs sujets d'exposés (Music Archives, ou groupes de discussion sur l'organisation du Cyberspace.)

Le développement de l'Internet, et de façon plus générale, des inforoutes ou autoroutes de l'information lancées il y a quelques années par le Vice Président Al Gore, et qui fait l'objet du récent sommet du G7 à Bruxelles pose un certain nombre de questions. Il y a bien sûr débat entre ceux qui pensent que l'on va vers une vision plus démocratique de l'accès au savoir puisque ce dernier est désormais accessible par tous à partir d'un réseau international. Certains se demandent en revanche si une sorte d'élite « technobranchée » n'est pas en train de se constituer, si l'on ne se dirige pas vers une ségrégation entre ceux qui auront accès à ces réseaux et sauront les utiliser facilement, et les autres, guettés par l'illettrisme électronique ${ }^{10}$.

71 La réponse est sans doute qu'à terme, et à très court terme, avec le tout numérique, en particulier la télévision numérique ${ }^{11}$, ces disparités tendront à s'effacer très vite. 
Lorsqu'on constate que l'université électronique reçoit 3000 demandes par jour, ce chiffre et cette évolution semblent être suffisamment parlants. L'Internet apparaît d'ores et déjà comme un instrument majeur d'échanges. L'impact sur la communauté scientifique est considérable, témoin la diffusion par courrier électronique du message du Dr. Thomas $\mathrm{R}$. Nicely, Lynchburg College, Virginia, qui découvrit les problèmes causés par le défaut de fabrication des microprocesseurs Pentium: diffusion très rapide et très vaste en même temps, qui eut les répercussions que l'on sait dans le monde industriel et boursier. Il est bien connu aussi que durant le putsch en ex-Union Soviétique le seul lien qui existait avec l'Ouest pendant un certain laps de temps fut l'Internet.

L'utilisation du courrier électronique, la participation à des groupes de discussion modifie la nature des échanges entre chercheurs ${ }^{12}$. Des serveurs comme I'M EUROPE, ou encore la banque de données CORDIS mis en place par la Commission Européenne contribuent aussi à une large diffusion auprès des chercheurs des appels d'offres de $1 \mathrm{a} \mathrm{CE}$ en matière de projets de recherche européens.

La diffusion de contenus culturels est importante et les contenus francophones sur l'Internet seront renforcés.

Les problèmes de sécurité sont bien sûr présents, mais 1a nature même des informations ${ }^{13}$ fait que les problèmes n'ont pas encore été très menaçants malgré leur fréquence : 1517 incidents sont signalés de janvier à septembre 94 par le Computer Emergency Response Team (CERT, Pittsburgh, Pennsylvania.) Le plus souvent, il s'agit du phénomène baptisé flaming, qui consiste à inonder la boîte aux lettres électronique de quelqu'un de centaines de messages (parfois insultants) ce qui bloque le système. Le problème de confidentialité des informations n'est bien sûr pas résolu pour autant, le Clipper chip qui aurait permis au FBI d'intercepter les messages a provoqué un tollé, et le débat sur les libertés à l'heure du numérique ne fait que commencer.

Quant au coût, il est supporté par les organismes de recherche pour une part et subventionné pour l'instant par les conseils régionaux pour encourager les universités à en faire usage ${ }^{14}$.

Pour les particuliers, des services commerciaux existent maintenant, à partir desquels on peut être relié au réseau par un modem, depuis chez soi. Les coûts sont certes assez élevés, mais pour un chercheur qui évite la perte de temps et le coût du transport pour l'accès à des données très éloignées géographiquement, cela est bien souvent utile. Ces services commerciaux connaissent une croissance très rapide. Les serveurs les plus connus sont Compuserve (9000 abonnés en France, 38 \% du marché aux États-Unis), Prodigy (38 \% aux USA) America on line, (17 \% aux USA), Delphi et Genie (3\% chacun). Pour la France, on peut également mentionner CalvaCom. Microsoft annonce son propre réseau (The Microsoft Network). Pour sa part, IBM propose déjà, avec le système d'exploitation 32 bits WARP et un modem, une possibilité de connexion à Internet (Bonus Pack) et c'est une initiative qui devrait se généraliser. Les prix sont très séduisants (trois premières heures de connexion gratuites, puis $90 \mathrm{FF}$ par mois pour trois heures de connexion et $25 \mathrm{FF}$ par heure supplémentaire) ${ }^{15}$. Apple pour sa part a lancé le service EWorld, pour simplifier l'accès aux services. Une série d'icônes (Arts and leisure pavilion, learning center, computer center, business and finance piazza, community centre, Email center, newsstand, info booth) permet d'effectuer un premier tri dans la masse d'informations.

77 À l'avenir, et pour nous universitaires, les subventions auront tendance à diminuer. Il est logique de prévoir de plus en plus de services payants, à l'instar de ce qui se passe pour 
les banques de données, l'accès rapide à l'information étant de plus en plus vital. La bataille des appétits commerciaux qui s'engage peut aussi à terme tourner à l'avantage du consommateur en provoquant une baisse des prix.

\section{Conclusion}

78 Pour ce qui est des langues, les enseignants ont, avec Internet, un outil puissant à leur disposition, que ce soit pour leur recherche ou pour leur enseignement. Des expériences ont déjà eu lieu dans le secondaire, en particulier les tentatives coordonnées par l'académie de Dijon, de mise en place d'un réseau pédagogique s'appuyant sur les outils multimédias pour huit académies pilotes avant une extension possible pour toute la France. Le lycée du Futuroscope de Poitiers expérimente ainsi une connexion bihebdomadaire avec l'Internet.

Les étudiants de langue sont motivés par l'utilisation de l'Internet qui est un formidable instrument de découverte de la langue, et d'une certaine manière, de la culture. La très grande variété des textes disponibles est particulièrement intéressante, et si on a cité ici des références à des écrits de type académique, on ne peut ignorer la production des particuliers dans les groupes de discussion. À la limite de l'écrit et du parler, ou parfois de très haut niveau comme les textes qui paraissent dans The Well (Sausolito, Californie) dont certains extraits ont été publiés par The Wall Street Journal, ou le New York Times. Certains affirment qu'une sorte de culture de l'Internet est en train d'émerger avec un style particulier, court, humoristique, inventif, percutant avec ses acronymes, et ses signes particuliers, formant un ensemble communément appelé Netiquette ${ }^{16}$.

Bien sûr, l'étudiant ne trouvera pas nécessairement, en tout cas point pour l'instant, un enseignement de la langue en tant que tel en ayant recours à l'Internet. Et reste à définir les mécanismes cognitifs qui présideront à l'apprentissage qu'il pourra ainsi faire de 1a langue. Mais s'il y a profusion de l'information, il y a aussi cheminements différents pour $\mathrm{y}$ avoir accès, et c'est peut-être aussi en cela que l'on peut parler d'Exploration Assistée par Ordinateur, assez éloignée de l'EAO traditionnel. L'apprenant sera invité à une certaine immersion, à une navigation où il sera maître de son parcours, même si l'on envisage une navigation guidée par un réseau de liens préétablis pour éviter les pertes de temps. Dans une acception un peu différente, on se rapproche du concept proposé par D. Wolff en 1993, de « l'apprenant en tant que chercheur ».

81 À terme, il n'est nullement irréaliste d'envisager une formation de langue directement accessible par l'Internet. Des projets européens sont d'ailleurs orientés en ce sens, et les exigences de formation de masse seront sans doute un des éléments qui encourageront les entreprises aussi à y avoir recours.

$82 \mathrm{Si}$ on veut faire un peu de prospective, on peut également penser que la notion d'apprentissage elle-même prendra une connotation différente. L'apprenant sera appelé à devenir lui-même un organisateur et un gestionnaire d'informations : c'est donc à nous de le former à ce nouveau rôle, donc a fortiori, de nous y former.

Je tiens à remercier l'UFR Informatique de Gestion pour les moyens techniques mis à ma disposition, ainsi que MM. Charmette, A. Fontaine et O. Gostan pour l'aide qu'ils m'ont apportée. 


\section{BIBLIOGRAPHIE}

Dieter Wolff. 1994. « Computers in classroom research ». Computers \& Education 23/1-2, 133-142.

Le Monde de l'Education. 1994. Novembre p. 55.

Séminaire. 1994. « Training in the Information Society ». 6-7 déc. 1994, Commission Européenne, DG XIII.

\section{ANNEXES}

\section{Ouvrages sur l'Internet}

Dern, D. P. 1993. The Internet Guide for New Users. Mc Graw-Hill.

Engst, A. 1993. The Internet Starter Kit for the Macintosh/ Hayden Books.

Moore, M. et al. 1994. The Internet Unleashed. Sams Publishing.

Fraase, M. 1993. PC Internet Tour Guide. Ventana Press.

Krol, E. 1993. The Whole Internet User's Guide and Catalog. O’Reilly \& Associates.

Kehoe, B. 1994. Zen and the Art of the Internet. Prentice Hall.

Les ouvrages français se mettent à fleurir. Pour une introduction, voir la collection ... pour les nuls. Le plus connu reste l'ouvrage de O’Reilly

2. Directement en ligne, que ce soit sur WWW, G N Navigator, XMOSAIC, ou NETSCAPE, des aides très détaillées sont disponibles:

-Entering the World Wide Web: A Guide to Cyberspace, (by Kevin Hughes, Enterprise Integration Technologies), May 94.

-Welcome to NCSA Mosaic... (inclut NCSA MOSAIC demo document, NCSA, 1994)

-GNN home page: Charting the Internet (O'Reilly and Associates, 1994)

-Welcome to the MOSAIC Communications Universe: NETSCAPE Guided Tour/NETSCAPE Handbook, Mosaic Communications Corporation, 1994)

On trouve également en ligne un glossaire sur Hypertexte/ hypermédia, et une histoire de l'hypermedia (dates principales).

3. The Whole Internet catalogue (sur GNN) liste les divers services. Voir aussi : Internet Resources list http://www.eit.com/web:netservices.html

4. Pour des informations spécialisées (Humanities/Language Learning), contacter :

-CTI Centre for Textual Studies, Oxford, qui a publié un Resources Guide, (Lorna Hughes \& Stuart Lee, OUCS, 1994). Voir en particulier les pp.3-6. Email: CTTTEXT@VAX.OX.AC.UK 
-CTI Centre for Modern Languages, Hull, qui publie régulièrement dans sa ReCALL

Newsletter, les adresses susceptibles d'être intéressantes pour les langues. Email:

CTI.Lang@hull.ac.uk

ReCALL Newsletter est maintenant consultable en ligne. Le serveur est: http:// www.hull.ac.uk/Hull/ITTI/homepage.html

\section{Quelques exemples}

Outils: TACT 2.1:gopher.epas.utoronto.ca, Cyrillic fonts:sumex-aim.stanford.edu, Logiciels SUMEXAIM.STANFORD.EDU

Données pour logiciels pédagogiques CELIA Computer Enhanced Language Instruction Archive gopher.archive.merit.edu, gopher.latrobe.edu.au, ftp at umich archive.umich.edu (directory CELIA FTP)

\section{NOTES}

1. Serveur MIAGE-Dauphine. Adresse: Erreur! Référence de lien hypertexte non valide. Réalisation informatique: A. Fontaine. Les informations contenues dans les pages du serveur n'engagent que leurs auteurs et en aucun cas l'Université ou son personnel.

2. Mary Mallery, «Overview of 'The Directory of Electronic Text Centers », Computers and Texts, 8, Dec. 1994, pp.2-3.

3. Renseignements auprès du Professeur David Bickerton, directeur du centre de langues de l'université de Plymouth (GB).

4. Voir annexe pour les adresses.

5. Voir, pour plus de détails sur les bibliothèques, les publications de L'URFIST de Paris (École des Chartes, 17, rue des Bernardins, 75005 Paris, téléphone : 432685 22.), en particulier « RENATER, Miniguide pour débutants, » qui est utile lorsqu'on ne dispose pas d'outils de type MOSAIC et que l'on travaille à partir d'une station UNIX. Nombreuses références sur les bibliothèques (listes de groupes de discussion, catalogues de bibliothèques.)

6. San Francisco's Exploratorium, The Krannert Art Museum, University of Califormia Museum of Paleontology, EXPO, Collaborative Art, International, Interactive, Genetic Art.

7. US Copyright Act, US patent Act, Uniform commercial code...

8. Maastricht Treaty, NATO Press Release, Privacy Act of 1974, United Nations Resolutions, Brady Handgun Violence Prevention Act, etc.

9. «The Visible Man on the Internet », International Herald Tribune, 17 Dec. 1994, p.9. Projet de la National Library of Medicine. «Images of the body were recorded from head to toe using computed tomography, magnetic resonance and X-rays. The body was then embedded in gelatin, frozen, and sliced crosswise. The slices were removed one by one and the surfaces photographed. The digital photos were then stacked and programmed into an imaging computer along with the data from the radiology images. [...] It is the first time such detailed digital information about an entire human body has been compiled. "

10. L'article de Jacques Buoh, "Le Cyberspace, enfer et paradis », Le Monde, 8 mars 1995, p.12, évoque les possibilités de dérapage de tels réseaux (terrorisme, mafia, etc.).

11. Canal + prévoyait de commercialiser des décodeurs numériques en octobre 1995 lorsque la chaîne offrira ses premiers services numériques. Canal + a déjà réservé sur ASTRA $1 \mathrm{E}$ et $1 \mathrm{~F}$ pour un développement à l'échelle européenne, et le numérique doit se développer sur le satellite TELECOM 1. De plus, La Cinquième étudie la possibilité de diffusion satellite en numérique qui faciliterait l'émission de programmes spécialisés destinés à des universités européennes. 
12. À un niveau plus microcosmique, le courrier électronique permet aussi aux étudiants de trouver des stages à l'étranger, en particulier dans les universités et laboratoires de recherche américains; il permet de gérer en ligne les échanges de correspondance de manière rapide et efficace.

13. Le serveur INTERNET de la CIA par exemple n'ouvre aucune porte aux informations confidentielles. Mais un autre réseau existe, INTELNET, fortement protégé. Il existe aussi des systèmes qui permettent de crypter les informations sensibles (Pretty Good Privacy, PGP), de manière assez sûre, même si les cypher punks s'essaient inlassablement au décryptage de ces données.

14. Voir le tableau des coûts RENATER en annexe. Communiqué par C. Panigel, URFIST de Paris, que je remercie vivement.

15. Alors que la tranche horaire est habituellement à $80 \mathrm{FF}$ et les abonnements à plus de $300 \mathrm{FF}$. Le magazine PC Direct de mai 1995 présente un tableau comparatif des différents prestataires de services en France. Emmanuel Parody, «Internet : tous les coûts sont permis », no.30, 138-157.

16. Par exemple : LOL : Laughing out Loud, IMO : In My Opinion, MOTOS : Members of the Opposite Sex, FAQ : Frequently Asked Question, KHYF : Know How You Feel, ou des contractions qui s'apparentent à la phonétique comme "CU” pour see you, ou «@@“pour indiquer une exclamation. Des signes particuliers existent aussi à base de signes de ponctuation (appelés emoticons ou smileys), imitant graphiquement les traits du visage, comme :-) pour un sourire, ou :-( pour indiquer la désapprobation, ou encore :-D pour le rire.

\section{RÉSUMÉS}

Cette étude est une introduction à l'utilisation de l'Internet. Elle décrit les différents outils de navigation ainsi que leur fonctionnement et dresse une typologie des sites Web dont le contenu peut se révéler utile pour l'enseignement de la langue et pour la recherche.

This paper is a guided tour of the Internet. It describes the different browsers that are now available and lists a number of Web sites whose resources can be used for language learning and research purposes.

\section{INDEX}

Mots-clés : forum, hypertexte, Internet, outil de navigation, World Wide Web

Keywords : browser, hypertext

\section{AUTEUR}

\section{NICOLE CHENIK}

Nicole Chenik est maître de conférences d'anglais à l'Université Paris 9 Dauphine. Son domaine de recherche est les Nouvelles Technologies de l'information et plus particulièrement leur utilisation dans l'apprentissage des langues et la recherche. Elle est l'auteur d'une thèse sur l'EAO 
et son application à l'enseignement de l'anglais de spécialité, ainsi que de divers articles publiés dans Ephémera, La Lettre du CLEO, Les Langues modernes, La Lettre du GERAS, ASp, System, et aux

Presses Universitaires de La Sorbonne. Elle est membre de l'association EuroCALL et fait partie du comité de lecture de la revue européenne ReCALL. Nicole.Chenik@dauphine.fr 